Communications in Physics, Vol.21, No. 4 (2011), pp. 309-320

\title{
ELECTRON MOBILITY IN AN UNINTENTIONALLY DOPED GaN/AlGaN SURFACE QUANTUM WELL
}

\author{
NGUYEN VIET MINH \\ Computational Physics Department, Institute of Engineering Physics, \\ Hanoi University of Science and Technology
}

\begin{abstract}
We present a theoretical study the two-dimensional electron gas 2DEG at low temperature in an unintentionally doped GaN/AlGaN surface quantum well, taking adequate account of the roughness-induced scattering mechansms and effect due to sheet polarization charges. Within model of surface quantum wells 2DEG be described by an extended Fang-Howard wave function, we are able to derive an analytic expression for the self-consistent Hartree potential. Thus, we obtained simple expresion describing the enhancement of the 2DEG screening and unscreened potentials for different scattering sources. We studied the electron mobility due to different scattering sources and the total electron mobility in an unintentionally doped GaN/AlGaN surface quantum well.
\end{abstract}

\section{INTRODUCTION}

Recently, experimental reports indicated that a two-dimensional electron gas (2DEG) is formed at the naked surface of several semiconductors, such as $\mathrm{ZnO}$ [1-4], SiGe [5] and GaN [6]. This open structure is referred to as a surface quantum well (SFQW) [7], in which a very high potential barrier $(\sim 4.5 \mathrm{eV})$ between the vacuum and the host crystal leads to an enhanced carrier confinement, i.e., a strong lateral quantization. In addition another surface quantum wells, where the vacuum leven acts as one of confining potentials and a wider band-gap heterojunction is the other confining potential was first demonstrated in the GaInP and GaInAs systems in 1987, and 1988, respectively $[8,9]$.

An understanding of SFQW is obviously important also for the modeling of lateral quantization in other open system, e.g, quantum wires and quantum dots. However, it should be mentioned that SFQW have been much less studied than quantum wells (QWs) [10].

Group-III-nitride-based heterostructures, in particular, the GaN/AlGaN have become technologically important for fabrication of high-voltage, high-power, and high temperature microwave devices. The electron mobility is an important transport parameter used to characterize the performance of these devices.

Thus, this paper is devoted to the development of a theory for the mobility of the two-dimensional electron gas 2DEG at low temperature in an unintentionally doped GaN/AlGaN surface quantum well. 


\section{TWO DIMENSIONAL ELECTRON GAS IN UID GaN/AIGaN SFQW}

In what follow, we will be dealing with a UID GaN/AlGaN SFQW. The crystal reference system is that the $\mathrm{z}$ axis is directed from vacuum to the well, and $z=0$ defines the plane between the vacuum $(z<0)$ and the GaN well layer $(z>0)$. It is assumed that the GaN layer be under tensile strain, while the AlGaN layer be relaxed. The electrons are confined in a QW separated from the vacuum by two potential barriers: one at $z<0$ and another at $z=L$, with of well. The barrier height between the vacuum and GaN is very large $\left(V_{0} \sim 5 \mathrm{eV}\right)[6]$, so that the penetration of electrons in to the vacuum is negligible. Due to surface roughness in both barriers, the realistic barriers are not absolutely flat interface. viz. the well width $\mathrm{L}$ is not constant. Therefore, the 2DEG in the lowest subband of a GaN/AlGaN SFQW is described by a modified Fang-Howard wave function, proposed by Ando [11,12]:

$$
\zeta(z)=\left\{\begin{array}{lc}
0 & \text { if } \quad z<0 \\
B k^{3 / 2} z e^{-k z / 2} & 0<z<L \\
A \kappa^{1 / 2} e^{-\kappa(z-L)} & z>L
\end{array}\right.
$$

in which $A, B, k, \kappa$ are variational parameters to be determined. Here $k$ and $\kappa$ are half the wave numbers in the well and barrier, respectively. $A, B$ are dimensionless parameters. Variational parameters $A, B, \kappa$ and the wave function (1) are determined by $k$ through boundary conditions at $z=L$, and the normalization one. And then, $k$ is determined by condition, in which the wave function (1) is to minimize the total energy per particle, which is determined by the Hamiltonian:

$$
H=T+V_{\text {tot }}(z)
$$

where $T$ is the kinetic energy, and $V_{\text {tot }}(z)$ is the total effective confining potential [13]:

$$
V_{\text {tot }}(z)=V_{\mathrm{w}}(z)+V_{b}(z)+V_{H}(z)+V_{i m}(z)+V_{\sigma}(z)+V_{\mathrm{xc}}(z),
$$

in which $V_{\mathrm{w}}(\mathrm{z})$ and $V_{b}(\mathrm{z})$ are the barrier potentials at $z=0$, and $z=L$, the third term $V_{H}(\mathrm{z})$ is the Hartree potential due to ionized dopants and confined electrons themselves. This is obtained from Poisson's equation

$$
\frac{d^{2}}{d z^{2}} V_{H}(z)=\frac{4 \pi e^{2}}{\varepsilon_{L}}\left[N_{D}(z)-n(z)\right]
$$

where $\varepsilon_{L}$ is the dielectric constant of sample, neglecting a small difference in its values between the layers with the use of an average value. The bulk density of ionized dopants in UID are given by

$$
N_{d}(z)=\left\{\begin{array}{lll}
0 & \text { for } \quad z<0 \text { and } z>z_{d} \\
N_{d} & \text { for } \quad 0<z<z_{d}
\end{array}\right.
$$

here, $z_{d}$ is UID thickness, $\mathrm{n}(\mathrm{z})$ is the bulk density of electron respectively. The electron density distribution is specified by the wave function from Eq. (1)

$$
n(z)=n_{s}|\zeta(z)|^{2}
$$


where $n_{s}$ is a sheet density of electron. Under overall charge neutrality condition [14], it hold

$$
n_{s}=N_{d} z_{d}
$$

We solve the Poisson's equation (4) with a boundery conditions that electric field corresponding to the Hartree is vanishing at infinity [14]. As a result, this potential is found as a sum

$$
V_{H}(z)=V_{I}(z)+V_{s}(z)
$$

in which the terms are to be regarded as the partial potentials created by the ionized dopants and the 2DEG.

The fourth term in Eq. (3) is the potential due to image charge, which quantities the effect arising from an abrupt decrease in the electric constant across the surface $z=0$. This is given by [15]

where by definition

$$
V_{i m}(z)=\frac{\varepsilon_{-}}{\varepsilon_{+}} \frac{e^{2}}{\varepsilon_{L}} \frac{1}{4 z}
$$

$$
\varepsilon_{ \pm}=\frac{\varepsilon_{L} \pm 1}{2 \varepsilon_{L}}, \quad \varepsilon_{+}+\varepsilon_{-}=1 .
$$

The fifth term in Eq. (3) is the potential due to spontaneous polarization charges bound on the AlGaN surface $(z=L)$, so that

$$
V_{\sigma}=\frac{2 \pi e^{2}\left(\sigma_{p} / e\right)}{\varepsilon_{L}} z
$$

with $\sigma_{p} / e$ as their sheet density.

At last, the exchange-correlation corrections allow for the many-body effect in the 2DEG along the normal direction. In the literature this was described by various models. Within a simplest model this is given by [16]

$$
V_{\mathrm{xc}}(z)=-0.611 \frac{e^{2}}{\varepsilon_{\mathrm{L}}}\left[\frac{3}{4 \pi} n(z)\right]^{1 / 3},
$$

with $n(z)$ as the electron distribution from Eq. (7).

\section{THE ELECTRON MOBILITY AT LOW TEMPERATURE}

The electron mobility at very low temperature may be determined within the relaxation time approximation by

$$
\mu=e \tau / m^{*}
$$

with $m^{*}$ as the in-plane effective electron mass of the GaN [17]. The inverse relaxation time for zero temperature is then expressed in terms of the autocorrelation function for each disorder [12]:

$$
\frac{1}{\tau}=\frac{1}{2 \pi \hbar E_{F}} \int_{0}^{2 k_{F}} d q \frac{q^{2}}{\left(4 k_{F}^{2}-q^{2}\right)^{1 / 2}} \frac{\left\langle|U(\mathbf{q})|^{2}\right\rangle}{\varepsilon^{2}(q)},
$$

where $q=2 k_{F} \sin (\theta / 2)$ as the $2 \mathrm{D}$ momentum transfer by a scattering event in the $x-y$ plane, with $\theta$ as a scattering angle. The Fermi energy is given by $E_{F}=\hbar^{2} k_{F}^{2} / 2 m^{*}$, with 
$k_{F}$ as the Fermi wave number fixed by the 2DEG density: $k_{F}=\sqrt{2 \pi n_{s}} \cdot\left\langle|U(\mathbf{q})|^{2}\right\rangle$ is autocorrelation function in wave vector space, that is specified for the different random scattering fields. Hereafter, the angular brackets stand for an ensemble average. $U(\mathbf{q})$ is a 2D Fourier transform of the unscreened scattering potential averaged with the envelope wave function of a $2 \mathrm{D}$ subband. The dielectric function $\varepsilon(q)$ entering in Eq. (14) takes account of the screening of a scattering potential by the 2DEG. As usual, this is evaluated within the random phase approximation [15]

$$
\varepsilon(q)=1+\frac{q_{s}}{q} F_{S}(q)[1-G(q)], \quad \text { for } q \leq 2 k_{F},
$$

where the inverse 2D Thomas-Fermi screening length is

$$
q_{s}=\frac{2 m^{*} e^{2}}{\varepsilon_{L} \varepsilon_{+} \hbar^{2}},
$$

We introduced the dimensionless wave numbers:

$$
t=q L, \quad a=k L, \quad \text { and } \quad b=\kappa L .
$$

The screening form factor $F_{S}(q)$ takes account of the extension of electronic states along the normal direction. With the wave function from Eq. (1), we obtained:

$$
\begin{aligned}
F_{S}(t)= & \varepsilon\left\{\left[\frac { 1 } { 2 } \left(\frac { 1 } { 2 L } \left(\frac{A^{4} b L(3 b-t)}{b^{2}-t^{2}}+\frac{a B^{4} e^{-2(a+t)} L\left(e^{2 a+t}(a-t)^{3}\left(8 a^{2}+9 a t+3 t^{2}\right)\right.}{\left(a^{2}-t^{2}\right)^{3}}\right.\right.\right.\right. \\
& -8 a^{5} e^{a}\left(2+a^{2}+2 t+t^{2}+2 a(1+t)\right)+e^{t}(a+t)^{3}\left(2 a^{6}-4 a^{5}(t-2)+3 t^{2}\right. \\
& \left.\left.\left.+3 a t(2 t-3)+2 a^{4}\left(8-6 t+t^{2}\right)+2 a^{3}\left(8-9 t+2 t^{2}\right)+2 a^{2}\left(4-9 t+3 t^{2}\right)\right)\right)\right) \\
& +a^{3} A^{2} b B^{2} e^{-a}\left\{\frac{2+a^{2}+2 b+b^{2}+2 a(1+b)-2 e^{a+b}}{(a+b)^{3}(b-t)}\right. \\
& +\frac{2+a^{2}-2 e^{a+t}+2 t+t^{2}+2 a(1+t)}{(a+t)^{3}(t-b)} \\
& +\frac{e^{-t}\left(-2 e^{a}+e^{t}\left(2+a^{2}-2 a(t-1)-2 t+t^{2}\right)\right)}{(a+b)^{3}(b-t)} \\
& -\left(\frac{1}{(a+b)^{3}(a-t)^{3}\left(t^{2}-b^{2}\right)}\right)\left[e ^ { - t } \left(2(a+b)^{3} e^{a}(b-t)+e^{t}(b+t)\left(4 t^{3}\right.\right.\right. \\
& +4 b(-1+t) t^{2}-a^{5}+a^{4}(4 t-3 b-2)-b^{3}\left(2-2 t+t^{2}\right) \\
& +2 b^{2} t\left(2-2 t+t^{2}\right)-a^{3}\left(2+3 b^{2}+b(6-10 t)-10 t+5 t^{2}\right) \\
& +a\left(2 b^{3}(t-1)+4(t-3) t^{2}-b^{2}\left(6-10 t+7 t^{2}\right)+4 b t\left(3-4 t+t^{2}\right)\right) \\
& \left.\left.\left.\left.\left.-a^{2}\left(b^{3}+b^{2}(6-8 t)-2 t\left(6-6 t+t^{2}\right)+b\left(6-18 t+11 t^{2}\right)\right]\right\}\right)\right)\right]\right\} \\
& +\varepsilon-\left\{\frac{B^{4} e^{-2(a+t)} a^{6}\left(2-2 e^{a+t}+(a+t)(2+(a+t))\right)^{2}}{2(a+t)^{6}}-\frac{A^{4} b^{2} e^{-2 t}}{2(t+b)^{2}}\right\},
\end{aligned}
$$


with $\varepsilon_{ \pm}$defined in Eq. (10). Here, the first $\left(\propto \varepsilon_{+}\right)$and second $\left(\propto \varepsilon_{-}\right)$terms are connected with the Coulomb interactions between the electrons and between them and their mirror images, respectively. The local field corrections are due to a many-body exchange effect in the 2DEG in the in-plane, given by [18]:

$$
G(t)=\frac{t}{2\left(t^{2}+t_{F}^{2}\right)^{1 / 2}} .
$$

At very low temperature the phonon scattering is negligibly weak. Therefore the electrons are expected to experience the following scattering sources: i) ionized dopants (ID), ii) alloy disorder (AD), iii) surface roughness (SR), iv) roughness-induced piezoelectric charges (PE) and v) roughness-induced deformation potential (DP). The total relaxation time is then determined by the ones for individual disorder according to Matthiessen's rule:

$$
\frac{1}{\tau_{\text {tot }}}=\frac{1}{\tau_{I D}}+\frac{1}{\tau_{A D}}+\frac{1}{\tau_{P E}}+\frac{2}{\tau_{S R}}+\frac{2}{\tau_{D P}},
$$

where we introduced a factor of 2 in last two terms on the right-hand side to include the effects from both interfaces of the SFQW. Thus, according to Eq. (14) we ought to specify the autocorrelation function in wave vector space $\left\langle|U(\mathbf{q})|^{2}\right\rangle$ for these scattering sources.

\section{III.1. Inonized dopants}

The autocorrelation function for scattering by randomly distributed charged impurities is shown $[15,19]$ to be represented in the form

$$
\left\langle\left|U_{I D}(\mathbf{q})\right|^{2}\right\rangle=\left(\frac{2 \pi e^{2}}{\varepsilon_{L} q}\right)^{2} \int_{-\infty}^{+\infty} d z_{i} N_{I}\left(z_{i}\right) F_{R}^{2}\left(q, z_{i}\right) .
$$

Here, $N_{I}\left(z_{i}\right)$ is the three-dimensional impurity density, and for UID: $N_{I}\left(z_{i}\right)=N_{d}$ for $0<$ $z_{i}<z_{d}$, and is zero elsewhere. $F_{R}\left(q, z_{i}\right)$ denotes the form factor for a sheet of impurities located in the plane $z=z_{i}$ and accounts for the extention of the electron state along the grownth direction, given by

$$
F_{R}\left(q, z_{i}\right)=\int_{-\infty}^{+\infty} d z|\zeta(z)|^{2} e^{-q\left|z-z_{i}\right|}
$$

Nevertheless, it has been experimentally indicated that [20] the assumption of the random impurity distribution fails to be valid at high doping levels, and for the understanding of several observable properties of heavily doped semiconductor systems one has to allow for high-temperature ionic correlation. This is due to Coulomb interactions between the charged impurities in their diffusion during growth and tends to reduce the probability for large fluctuations in their density and, hence, in their potential, so reducing the autocorrelation function. Thus, the ionic correlation may be referred to as a statistical screening and weakens the impurity scattering, so increasing the respective partial mobility.

It was shown that [21] for taking into account the ionic correlation, we have to incorporate an appropriate correlation factor (less than unity) into the autocorrelation function as follows [12]

$$
\left\langle\left|U_{I D}(\mathbf{q})\right|^{2}\right\rangle_{c}=\left\langle\left|U_{I D}(\mathbf{q})\right|^{2}\right\rangle \frac{q}{q+q_{i}} .
$$


Here, the angular brackets with subindex c means the ensemble average over the correlated impurity distribution, and $q_{i}$ is inverse statistical screening radius, given by

$$
q_{i}=\frac{2 \pi e^{2} n_{d}}{\varepsilon_{L} k_{B} T_{0}}
$$

where $n_{d}=N_{d} L_{d}$ is the $2 \mathrm{D}$ impurity density, and $T_{0}$ the freezing temperature for impurity diffusion $(\sim 1000 \mathrm{~K})$.

With the use of lowest-subband wave function from Eq. (1), and the dimensionless wave numbers from Eq. (17), we may find the autocorrelation function for scattering by correlated ionized dopants in form of a special analytic function $f r 2 t(t)$ in [22] that is easy calculated by Mathematica software but can not be represented here

$$
\left\langle\left|U_{I D}(t)\right|^{2}\right\rangle_{c}=\left(\frac{2 \pi e^{2}}{\varepsilon_{L}}\right)^{2} n_{d}\left(\frac{t}{t+t_{i}}\right) \frac{L^{2} f r 2 t(t)}{t^{2}},
$$

here $t_{i}=q_{i} L$.

\section{III.2. Alloy disorder}

The autocorrelation function for scattering is supplied in form [11]

$$
\left\langle\left|U_{A D}(\mathbf{q})\right|^{2}\right\rangle=x(1-x) u_{a l}^{2} \Omega_{0} \int_{0}^{L} d z|\zeta(z)|^{4}
$$

in which, $x$ is the $\mathrm{Al}$ content, $u_{a l}$ is the alloy potential, $\mathrm{L}$ is the well width. The volume occupied by one alloy atom is given by $\Omega_{0}=a^{3}(x) / 8$, which $a(x)$ the lattice constant of the alloy [12].

By mean of Eq. (1) for the lowest-subband wave function and the dimensionless wave numbers from Eq. (17), the autocorrelation function for scattering by alloy disorder is written as follows:

$$
\left\langle\left|U_{A D}(t)\right|^{2}\right\rangle=x(1-x) u_{a l}^{2} \Omega_{0}\left\{\frac{1}{4} B^{4} \frac{a}{L}\left[3-e^{-2 a}\left(3+6 a+6 a^{2}+4 a^{3}+2 a^{4}\right)\right]\right\}
$$

\section{III.3. Surface roughness}

Here, we treat the scattering of 2DEG from a rough potential barriers, one at $z=0$ and another at $z=L$. It was pointed out [12] that the autocorrelation function for surface roughness scattering is fixed by the local value of the wave function at the surface:

$$
U_{S R}(\mathbf{q})=V_{0}|\zeta(0)|^{2} \Delta_{q}
$$

where $\Delta_{q}$ is surface rouhgness profile.

It is to be noticed that at $z=0$ the right-hand side of Eq. (28) becomes indefinite in the limiting case of infinite potential barrier $\left[V_{0} \rightarrow \infty\right.$ and $\left.\zeta(0) \rightarrow 0\right]$. Therefore, we need to adopt the following formula valid for any bound electronic state: [12]

$$
\int_{-\infty}^{+\infty} d z|\zeta(z)|^{2} \frac{\partial V_{\mathrm{tot}}(z)}{\partial z}=0
$$

which is exact and applicable for any value of the barrier height $V_{0}$. Upon replacing the effective confining potential with Eq. (3), we may represent the local value of the wave 
function via the expectation values of the electric fields created by the partial confining sources:

$$
V_{0}|\zeta(0)|^{2}=V_{0}|\zeta(L)|^{2}=\left\langle V_{H}(z)^{\prime}\right\rangle+\left\langle V_{i m}(z)^{\prime}\right\rangle+\left\langle V_{\sigma}(z)^{\prime}\right\rangle+\left\langle V_{\mathrm{xc}}^{\prime}\right\rangle,
$$

with $V^{\prime}=\partial V(z) / \partial z$.

Next, by putting Eq. (30) into Eq. (28), we obtain the autocorrelation function for surface roughness:

$$
\begin{aligned}
\left\langle\left|U_{S R}(q)\right|^{2}\right\rangle= & \left(\frac{2 \pi e^{2}}{\varepsilon_{L}}\right)^{2}\left[e^{-k L}\left(\frac{\sigma}{e}\right)\left(A^{2} e^{k L}+B^{2}\left(2-2 e^{k L}+2 k L+k^{2} L^{2}\right)\right)\right. \\
& -\frac{2 A^{2} N_{d}\left(e^{\left(L-z_{d}\right) k}-1-L k+z_{d} \kappa\right)}{\kappa}+\frac{n_{s}}{2} e^{-2 k L}\left(B ^ { 4 } \left(2-2 e^{k L}\right.\right. \\
& \left.\left.\left.+2 k L+k^{2} L^{2}\right)^{2}-A^{4} e^{2 k L}\right)\right)+0.015083\left(\frac{2 \pi e^{2}}{\varepsilon_{L}}\right) n_{s}^{1 / 3}\left(A^{8 / 3} \kappa^{4 / 3}\right. \\
& \left.\left.-B^{8 / 3} e^{-\frac{4 k L}{3}} k^{4} L^{8 / 3}\right)\right]^{2}\left\langle\left|\Delta_{\mathbf{q}}\right|^{2}\right\rangle
\end{aligned}
$$

As seen from Eq. (28), surface roughness scattering is specified by the surface profile. This is normally written as:

$$
\left\langle\left|\Delta_{\mathbf{q}}\right|^{2}\right\rangle=\pi \Delta^{2} \Lambda^{2} F_{S R}(t),
$$

where $\Delta$ is a roughness amplitude, and $\Lambda$ a correlation length. The roughness form factor is given by: [23]

$$
F_{S R}(t)=\frac{1}{\left(1+\lambda^{2} t^{2} / 4 n\right)^{n+1}},
$$

where $n$ is an exponent fixing its falloff at large momentum transfer in range $n=1$ to 4 , and $\lambda=\Lambda / \sigma \sqrt{2}$ a dimensionless correlation length.

\section{III.4. Roughness-induced piezoelectric charges}

In wurtzite III-nitride heterostructures, e.g. GaN/AlGaN, surface roughness gives rise to strain fluctuations in both strained and relaxed layers. In Ref. [24] Quang and coworkers have demonstrated that the strain fluctuations produce random nonuniform variations in the piezoelectric polarization. These in turn induce fluctuating densities of piezoelectric charges, viz. bulk charges of strained and relaxed layers as well as sheet charges on the interface. The charges create relevant electric fields and act as scattering sources on the $2 \mathrm{D}$ motion of electron in the in-plane. It has been pointed out [24] that the average electric field due to sheet charges is much weaker than those of bulk charges. In addition, the average field due to bulk charges in GaN well is nearly equal to that in the AlGaN barrier. Therefore, we may plausibly restrict ourselves to calculate the scattering by bulk charges located in the well layer.

The potential energy for an electron moving in the field due to roughness-induced bulk piezoelectric charges in the channel layer is described by [12]:

$$
U_{P E}(\mathbf{q}, z)=\frac{\pi \alpha \epsilon_{\|} e Q}{\varepsilon_{L}} q \Delta_{q} F_{P E}(q, z)
$$


Here, $\alpha$ denotes the anisotropy ratio as a measure for the deviation of hexagonal symmetry of the wurtzite crystal from isotropy, $\epsilon_{\|}$is the latice mismatch. $Q$ is a material parameter characteristic of the well, defined in term of its elastic stiffness $c_{i j}^{w}$ and piezoelectric $e_{i j}^{w}$ constants by [12].

$$
Q=\frac{C_{b}}{c_{33}^{b}}\left[\frac{e_{15}^{w}}{c_{44}^{w}}+\frac{e_{31}^{w}\left(c_{33}^{w}+2 c_{13}^{w}\right)-e_{33}^{w}\left(c_{11}^{w}+c_{12}^{w}+c_{13}^{w}\right)}{C_{w}}\right],
$$

with

$$
C_{\lambda}=c_{33}^{\lambda}\left(c_{11}^{\lambda}+c_{12}^{\lambda}\right)-2\left(c_{13}^{\lambda}\right)^{2},
$$

here $(\lambda=b, w)$ is noted for the barrier and well layers respectively.

The form factor in Eq. (34) is given by

$$
F_{P E}(q, z)=\frac{1}{2 q}\left\{\begin{array}{lc}
e^{q z} 2 q L & \text { for } z<0 \\
2 q z e^{q z}+2 e^{L q} \operatorname{Sinh}[q(L-z)] & 0<z<L \\
e^{-q z}\left(e^{2 L q}-1\right) & z>L .
\end{array}\right.
$$

Upon averaging Eqs. (34) and (37) by mean of the lowest subband wave function from Eq. (1), we obtained the weighted potential for scattering by roughness-induced piezoelectric charges

$$
U_{P E}(\mathbf{q})=\frac{\pi \alpha \epsilon_{\|} e Q}{\varepsilon_{L}} F_{P E}(q / k) \Delta_{q}
$$

The weighted piezoelectric form factor is expressed as a function of the dimensionless wave number in Eq. (17):

$$
\begin{array}{r}
F_{P E}(t)=\frac{1}{\left.(a-t)^{4}\right)}\left[a^{3} L B^{2}\left(6-e^{t-a}(6+(6+(a-t)(3+a-t))(a-t))\right)\right] \\
+\frac{A^{2} b L e^{-t}\left(e^{2 t}-1\right)}{2 t(b+t)}+\frac{1}{t\left(a^{2}-t^{2}\right)^{3}}\left[a ^ { 3 } B ^ { 2 } L e ^ { - a } \left(e^{a+2 t}(a-t)^{3}-e^{a} k^{3}(a+t)^{3}\right.\right. \\
\left.\left.+e^{t}\left(a^{2}(6+a(4+a)) t-2(a(2+a)-1) t^{3}+t^{6}\right)\right)\right]
\end{array}
$$

\section{III.5. Roughness-induced deformation potential}

Rougness-induced strain fluctuations give rise to random nonuniform shifts of band edges of the conduction and valence bands. This implies that electrons in the conduction band and holes in the valence one must experience a perturbating potential. The roughness-induced deformation potential for electron is determined by fluctuations of a diagonal strain component $\Delta \epsilon_{z z}$, according to [12]

$$
U_{D P}=\Xi_{d} \Delta \epsilon_{z z}
$$

where $\Xi_{d}$ is the combined dilational component of the deformation potential for conduction band. Since the deformation potential is of short range, and the 2DEG is located mainly in the well, we can reasonably take into account the relevant scattering merely in this layer. 
Follow [12], we get a 2D Fourier transform of the perturbating potential for the electron as follows:

$$
U_{D P}(\mathbf{q}, z)=\frac{\alpha \epsilon_{\|} \Xi_{d}}{2} \frac{C_{b}}{c_{33}^{b}} \frac{c_{11}^{w}+c_{12}^{w}+c_{13}^{w}}{C_{w}} q \Delta_{\mathbf{q}} e^{-q z} .
$$

for $0<z<L$, and is zero elsewhere. Here, $c_{i j}^{\lambda}$ are the elastic stiffness constants of the $\lambda$ layer $(\lambda=b, w)$, and $C_{\lambda}$ are determined from Eq. (36)

Upon averaging Eq. (36) by means of the lowest-subband wave function from Eq. (1). we may represent the autocorrelation function for deformation potential scattering in the form

$$
\left\langle\left|U_{D P}(\mathbf{q})\right|^{2}\right\rangle=\left\{\frac{\alpha \epsilon_{\|} \Xi_{d}}{2} \frac{C_{b}}{c_{33}^{b}} \frac{c_{11}^{w}+c_{12}^{w}+c_{13}^{w}}{C_{w}} q F_{D P}(q / k)\right\}^{2}\left\langle\left|\Delta_{\mathbf{q}}\right|^{2}\right\rangle .
$$

where the formfactor is given by

$$
F_{D P}(t)=\frac{A^{2} b e^{-t}}{b+t}+\frac{a^{3} B^{2}\left(2-e^{-(a+t)}\left[2+a^{2}+2 t+t^{2}+2 a(1+t)\right]\right)}{(a+t)^{3}} .
$$

\section{RESULTS AND CONCLUSIONS}

In this section, we are trying to apply the foregoing theory to understand transport properties of the 2DEG in wurtzite UID GaN/AlGAN SFQW. For numerical results, we have to specify parameters appearing in the theory as input. The lattice constant, elastic stiffness constants, piezoelectric constants, and dielectric constant for AlN and GaN are taken from Refs. $[12,25]$. The coresponding constants for an AlGaN alloy are estimated within the virtual crystal approximation [25]. The potential barrier heigh $V_{0}$ as usual, this is to be equal to the conduction band offset between the $\mathrm{Al}_{x} \mathrm{Ga}_{1-x} \mathrm{~N}$ barrier and the GaN well, which depend on the $\mathrm{Al}$ content $x$ as $[26,27]$ :

$$
\Delta E_{c}=0.75\left[E_{g}(x)-E_{g}(0)\right]
$$

where the band gap of $A l_{x} G a_{1-x} N$ is measure to be [25]:

$$
E_{g}(x)=6.13 x+3.42(1-x)-x(1-x) e V
$$

The anisotropy ratio $\alpha$ of the wurtzite $\mathrm{GaN}$ is chosen $\alpha=5$ [25] as a typical value in our numerical calculation. We have carried out numerical calculations of the low-temperature 2DEG mobility due to piezoelectric scattering in UID GaN/ $\mathrm{Al}_{0.25} \mathrm{Ga}_{0.75} \mathrm{~N}$ SFQW. The effective electron masses of GaN are for the growth direction $m_{z}=0.18 m_{e}$ [17] and for the in plane $\mathrm{m}^{*}=0.228 m_{e}[28]$. We carried out calculation the influence of density of sheet of polarization charge $\sigma / e$ on the wave function of 2DEG, and the total electron mobility as well as electron mobility due to different scattering sources versus sheet electron density $n_{s}$, and dependence of the electron mobility on an Al-content $x$. From calculation results obtained, we may draw the following conclusions:

i) Figure. 1 reveals that the variation of density of sheet of polarization charge $\sigma / e$ has influencing to distribution of 2DEG in well. When density of sheet of polarization charge is small $\left(\sigma / e<7.10^{12}\right)$, the positive potential that is created by positive polarization 


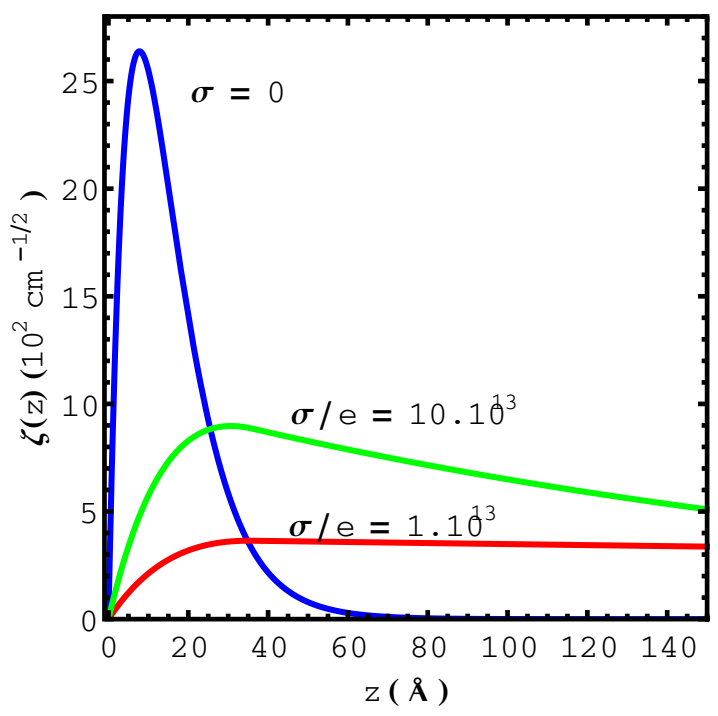

Fig. 1. The wave functions of $2 \mathrm{DEG}$ of an UID $G a N / A l_{0.25} G a_{0.75} N$ SFQW for different density of sheet of polarization charge $\sigma / e$.
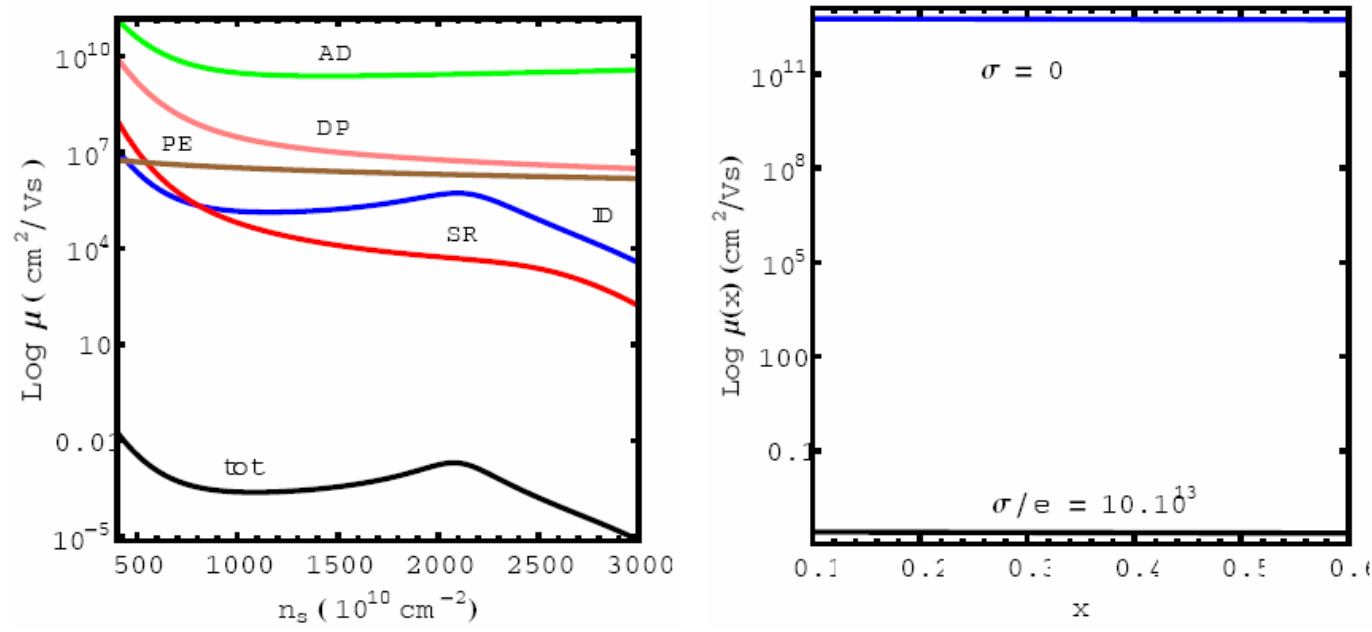

Fig. 2. 2DEG mobility of an UID GaN/AlGaN SFQW vs. sheet electron density $n_{s}$ and vs. an $\mathrm{Al}$ content $x$.

charges is not enough prevail negative potential of the well, therefore the 2DEG is located mainly in the well layer, and when density of sheet of polarization charge is enough large $\left(\sigma / e>7.10^{12}\right)$, the electric field that is created by positive polarization charges push the 2DEG to barrier at $z=L$ where is located polarization charges-the interface plane and when density of sheet of polarization charge is increased, the peak of electron distribution is raised, so the $2 \mathrm{DEG}$ is pushed closer to interface plane. 
ii) Figure 2 reveals that total electron mobility is small, and in the high-density regime of 2DEG surface roughness and ionized dopants are found to be predominant scattering sources over the other scattering sources: alloy disorder, deformation potential and piezoelectric charges.

iii) Figure 2 reveals that the electron mobility is reduced by polarization charges. The mobility reduction is due to that the polarization charges can cause an reduction of electron mobility in two ways. On the one hand, via the polarization confinement effect they facilitate the redistribution of $2 \mathrm{D}$ electrons in the interface plane, where are the key scattering sources, scattering decreased the electron mobility. On the another hand via their electric field they elevate the density of electrons, so enhancing the screened potential in each scattering potential, so reduced mobility.

To summarize, in this contribution we have theoretical studied the electron mobility of the two-dimensional electron gas in a UID GaN/AlGaN SFQW. We have derived analytic expression, which explicitly describe the scattering rates for different scattering process limiting the 2DEG. We have examined dependence of the total electron mobility, also each mobility due to different scattering sources on sheet electron density and on $\mathrm{Al}$ content. The presence of the polarization charges on profile plane decreased the electron mobility. The influence of polarization charges on the electron mobility will be studied in another our paper.

\section{ACKNOWLEDGMENT}

Financial support of Science and Technology Research program (project B2009-01268) is gratefully acknowledged.

\section{REFERENCES}

[1] D. Eger and Y. Goldstein, Phys. Rev. B19 (1979) 1089.

[2] Y. Grinshpan, M. Nitzan, and Y. Goldstein, Phys. Rev. B19 (1979) 1098.

[3] E. Veuhoff and D. Kohl, J. Phys. C14 (1981) 2395.

[4] G. Yaron, A. Many, and Y. Goldstein, J. Appl. Phys. 58 (1985) 3508.

[5] K. Kishimoto, Y. Shiraki, and S. Fucatsu, Thin Solid Films 81 (1998) 321. Appl. Phys. Lett. 70 (1997) 2837.

[6] J. F. Muth, X. Zhang, A. Cai, D. Fothergill, J. C. Roberts, P. Rajagopal, J. M. Cook, Jr. E. L. Piner, and K. J. Linthicum, Appl. Phys. Lett. 87 (2005) 192117.

[7] J. Lindhart, M. Scharff, and H. E. Schiott, Kong. Danske Vid. Selsk, Mat.-Fis. Medd. N.13 (1963) 33

[8] R. M. Cohen, M. Kitamura. and Z. M. Fang, Appl. Phys. Lett., 50 (1987) 1675.

[9] E. Yablonovitch, H. M. Cox, and T. J. Gmitter, Appl. Phys. Lett. 52 (1988) 1002.

[10] Doan Nhat Quang, Le Tuan, Nguyen Thanh Tien, Phys. Rev. B77 (2008) 125326.

[11] T. Ando, J. Phys. Soc. Jpn. 51 (1982) 3893;

[12] D. N. Quang, V. N. Tuoc, N. H. Tung, N. V. Minh, and P. N. Phong, Phys. Rev. B72 (2005) 245303.

[13] Nguyen Viet Minh, Physics and Engineering in Evolution, Science and Technics Publishing House, (2008) 13

[14] G. Bastard, Wave Mechanics Applied to Semiconductor Heterostructures (Les Editions de Physique, Paris, 1998

[15] T. Ando, A. B. Fowler, and F. Stern, Rev. Mod. Phys. 54 (1982) 437.

[16] W. Kohn and L. J. Sham, Phys. Rev. 140 (1965) A1133.

[17] L. W. Wong, S. J. Cai, R. Li, K. Wang, H. W. Jang, and M. Chen, Appl. Phys. Lett. 73 (1998) 1391. 
[18] M. Jonson, J. Phys. C, 9 (1976) 3055

[19] Gold, Phys. Rev. B 35, (1987)723

[20] F. Schubert, J. M. Kuo, R. F. Kopf, H. S. Luftman, L. C. Hopkins, and N. J. Sauer, . Appl. Phys. 67 (1990) 1969

[21] D. N. Quang and N. H. Tung, Phys. Status Solidi B207 (1998) 111.

[22] Nguyen Viet Minh, unpublished (2009).

[23] R. M. Feenstra and M. A. Lutz, J. Appl. Phys. 78 (1995) 6091.

[24] D. N. Quang, V. N. Tuoc, N. H. Tung, N. V. Minh, and P. N. Phong, Phys. Rev. B72 (2005) 115337.

[25] O. Ambacher, B. Foutz, J. Smart, J. R. Shealy, N. G. Weimann, K. Chu, M. Murphy, A. J. Sierakowski, W. J. Shaff, I. F. Eastman, R. Dimitrov, A. Mitchell, and M. Stutzmann, Appl. Phys. 87 (2000) 334.

[26] G. Martin, S. Strite, A. Botchkarev, A. Agarwal, A. Rockett, and H. Morkoc, Appl. Phys. Lett. 65 (1994) 610 .

[27] G. Martin, A. Botchkarev, A. Rockett, and H. Morkoc, Appl. Phys. Lett. 68 (1996) 2541.

[28] L. Hsu and W. Walukiewicz, J. Appl. Phys. 89 (2001) 1783.

Received 20 August 2010. 